\title{
Kinetic Magnetism and Orbital Order in Iron Telluride
}

\author{
Ari M. Turner, ${ }^{1}$ Fa Wang, ${ }^{1,2}$ and Ashvin Vishwanath ${ }^{1,2}$ \\ ${ }^{1}$ Department of Physics, University of California at Berkeley, Berkeley, CA 94720 \\ ${ }^{2}$ Materials Sciences Division, Lawrence Berkeley National Laboratory, Berkeley, CA 94720
}

(Dated: October 31, 2018)

\begin{abstract}
Iron telluride (FeTe), a relative of the iron based high temperature superconductors, displays unusual magnetic order and structural transitions. Here we explore the idea that strong correlations may play an important role in these materials. We argue that the unusual orders observed in FeTe can be understood from a picture of correlated local moments with orbital degeneracy, coupled to a small density of itinerant electrons. A component of the structural transition is attributed to orbital, rather than magnetic ordering, introducing a strongly anisotropic character to the system along the diagonal directions of the iron lattice. Double exchange interactions couple the diagonal chains leading to the observed ordering wavevector. The incommensurate order in samples with excess iron arises from electron doping in this scenario. The strong anisotropy of physical properties in the ordered phase should be detectable by transport in single domains. Predictions for ARPES, inelastic neutron scattering and hole/electron doping studies are also made.
\end{abstract}

PACS numbers:

\section{INTRODUCTION}

The discovery of high temperature superconductivity in a class of iron based materials ${ }^{1}$ has opened a new route to high temperature superconductivity besides the ones operating in the copper oxide materials. Following the initial discovery in LaOFeAs (1111 materials), a number of new classes of materials were discovered that shared similar properties, notably the 122 materials (like $\mathrm{BaFe}_{2} \mathrm{As}_{2}$ ). In these systems, (collectively referred to as the FeAs materials) the undoped compound is a metallic SDW system, with ordering wavevector $(\pi, 0)$, which on doping leads to a superconducting state. The magnetism in these materials is believed to arise from Fermi surface nesting, given the presence of an electron and hole pocket separated by $(\pi, 0)$ in the LDA band structure calculations of these materials. In fact, theoretical studies had predicted this ordering before it was confirmed in neutron scattering experiments. Moreover, the ordering moment is typically quite small, eg. of order $0.3 \mu \mathrm{B}$ in $\mathrm{LaOFeAs}$, and the absence of a Curie-Weiss form of magnetic susceptibility above the ordering temperature have been invoked as evidence for the itinerant character of the magnetism. Finally, signatures of an excitation gap appear in optical conductivity experiments, on cooling through the SDW transition $\underline{\underline{2}}$

An important recent development has been the discovery of a new class of materials FeSe and FeTe, which share the square lattice Fe structure of the FeAs materials and are believed to be closely related. Indeed, superconductivity has been observed in FeSe even in the absence of doping at $8 K$, rising to $37 \mathrm{~K}$ on application of hydrostatic pressure. The chemical simplicity of these materials, as well as the absence of a pnictide group element, may offer valuable clues to isolating the physics of the iron based high temperature superconductors. One notable difference from the FeAs materials though, is in the magnetism. While FeSe is non-magnetic even at sto- ichiometry, the FeTe materials are magnetically ordered metals, but with a more complicated kind of order than seen in FeAs, shown in Fig. 1. The ordering wavevector is $(\pi / 2, \pi / 2)$, in contrast to the $(\pi, 0)$ ordering of the FeAs compounds and also the $(\pi, \pi)$ ordering of the insulating parent compound of the cuprate superconductors. (Note the wavevectors here are defined with respect to an unfolded zone comprising of a unit cell of one iron atom, oriented along the iron square lattice. The actual basis vectors are $\hat{\mathbf{x}} \pm \hat{\mathbf{y}}$ because of the alternating positions of the tellurium ions; therefore, crystallographic studies use a doubled-unit cell, and $a$ and $b$ are along the diagonals of the iron lattice.) The order sets in via a first order transition at $87 K$, and is accompanied by a monoclinic distortion ${ }^{3}$. In the presence of excess iron, i.e. $F e_{1+y} T e$, the commensurate order is found to evolve into an incommensurate spiral. Whether this complex magnetic behavior is important to understanding other physics in this material is presently unclear. So far, it is unique to $\mathrm{FeTe}$, where superconductivity only occurs on substantial alloying with sulfur $F e S_{0.2} T e_{0.8}$ or selenium $\mathrm{FeSe}_{0.5} T e_{0.5}$. However, besides the interest in understanding the origin of this unusual magnetism, there are a number of indicators that point to the presence of strong correlations in $\mathrm{FeTe}$, which would be an important fact to establish in these materials. (1) The ordered magnetic moment in FeTe is large, $\sim 2 \mu_{B}$, consistent with a localized $S=1$ at every site. (2) Above the ordering temperature the magnetic susceptibility falls off in a Curie Weiss fashion, roughly consistent with the observed ordered moment and transition temperature. (3) given the absence of Fermi surface nesting at this wavevector, a spin density wave scenario seems less favorable than a local moment picture. Furthermore, ARPES experiments on this material see no obvious nesting at the desired wave-vector ${ }^{4}$, and (4) optical conductivity, which observes a clear SDW gap in the FeAs materials, does not see an analogous gap in $\mathrm{FeTe}^{\underline{5}}$. Another mechanism 


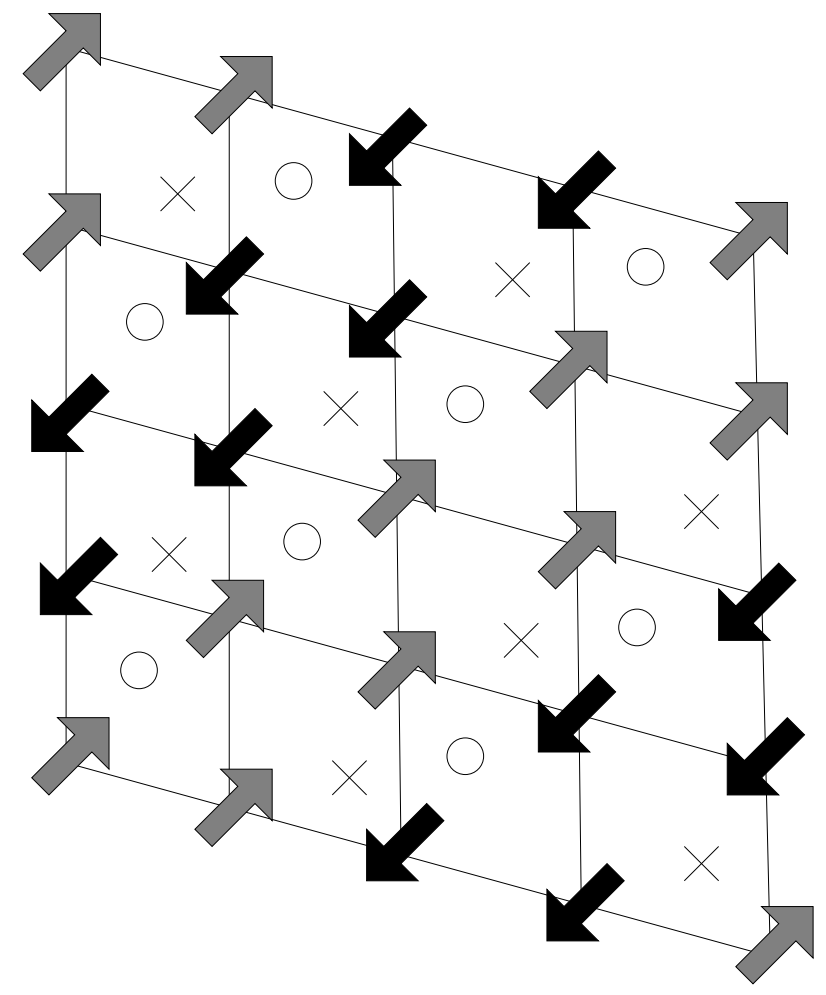

FIG. 1: The commensurate $\left(\frac{\pi}{2}, \frac{\pi}{2}\right)$ spin ordering pattern of $\mathrm{Fe}_{1+x} \mathrm{Te}_{1}$, close to $x=0$. The spins reside on the iron atoms, which form a square lattice. The tellurium atoms alternate above (x's) and below (o's) the iron plane. When the spins order, the square lattice of irons distorts into an approximately rhombic lattice. We assume that the monoclinic distortion is a consequence of the spin ordering. The tellurium atoms move towards (or away from) the exceptional spin in each parallelogram. Thus, the planes of tellurium atoms shift in opposite directions, producing the monoclinic distortion.

is therefore needed to explain the peculiar spin ordering. Here we will assume that the magnetism in FeTe arises from local magnetic moments on the iron sites, that arise from strong correlations. However, given that FeTe is metallic (with a residual resistivity of $0.2 \mathrm{~m} \Omega \mathrm{cm}$ ) - we will have to consider them as being 'self-doped'. The key point of this paper is that several of the puzzling magnetic properties of $\mathrm{FeTe}$ can be naturally explained if we assume it is near a correlated insulating state with spin and orbital degeneracies. Furthermore, the structural distortion here will be explained as arising, at least partially, from orbital ordering - rather than spin lattice interaction as is usually assumed. A model for FeTe's ordering in which the lattice distortion is assumed from the outset is in $\underline{\underline{6}}$. Other scenarios based on an itinerant electron picture have also been put forward ${ }^{7}$, such as the possibility that electron doping is large enough to change the Fermi surface shape and lead to nesting ${ }^{8}$.

The main assumptions we make in this paper are: (i) FeTe is proximate to an $S=1$ magnetic insulator. (ii)Each site of this insulator has orbital degeneracy $\left(d_{1}, d_{2}\right)$ and the Jahn Teller effect leads to orbital ordering which orients the orbitals along the diagonal direction of the Fe lattice. The orbital $d_{1}$ accommodating the local moment is uniformly oriented on all sites. (iii) A small excess density of charge carriers is present in the other Jahn Teller orbital $d_{2}$.

From these assumptions we show that the $(\pi / 2, \pi / 2)$ magnetic order can be naturally explained, as well as the incommensuration induced by excess iron. The key mechanisms are the formation of one dimensional chains induced by the Jahn-Teller ordering, which are coupled together by double exchange. Part of the structural transition in this scenario is caused by orbital rather than spin ordering. Since this proposal invokes an intertwining of the spin, charge and orbital physics, several testable consequences also emerge for transport and ARPES experiments. For example, transport within a single domain is expected to be highly anisotropic, with larger conductivity along the ferromagnetically ordered diagonal directions. This can potentially be probed by optical conductivity experiments. Indeed, a number of anomalies are already observed in transport properties, albeit in multidomain samples. The electrical conductivity rises sharply below the ordering transition while the Hall effect abruptly changes sign.

Recently, a similar scenario has appeared to explain the magnetism and structural transition in $\mathrm{FeAs}_{\mathrm{s}}$ 10,11. In contrast to our assumption (ii), the Jahn-Teller orbitals are assumed to order along the lattice directions. The spin wave spectrum experimentally observed in FeAs was argued to be much better described by such a model ${ }^{10}$. Here we focus on $\mathrm{FeTe}$, for the reasons described previously, but our approach is very similar in spirit. If indeed this mechanism is more general, it allows us to unify phenomena across this family of compounds.

This paper is organized as follows. We first describe microscopic strongly correlated models for FeTe, which have $\mathrm{S}=1$ and orbital degeneracy. We then consider how orbital ordering of a particular kind can drive magnetism, leading to the observed spin order. The characteristics of spin wave dispersion within this scenario are presented, and the origin of incommensurate magnetism with excess iron, is discussed. Finally, experimental consequences of this scenario for nonmagnetic properties, like conductivity and ARPES, are described.

\section{FeTe: A STRONGLY CORRELATED VIEWPOINT}

\section{A. Microscopic Model}

We first model FeTe in terms of a nearby correlated insulating state. The modifications required to account for metallic conduction are discussed later. We demand that the insulator carries net spin $S=1$, per Fe atom, based on the ordered moment observed at low temperatures. Furthermore, we will require that they exhibit orbital degeneracy. The only pair of $d$ orbitals that are degenerate 

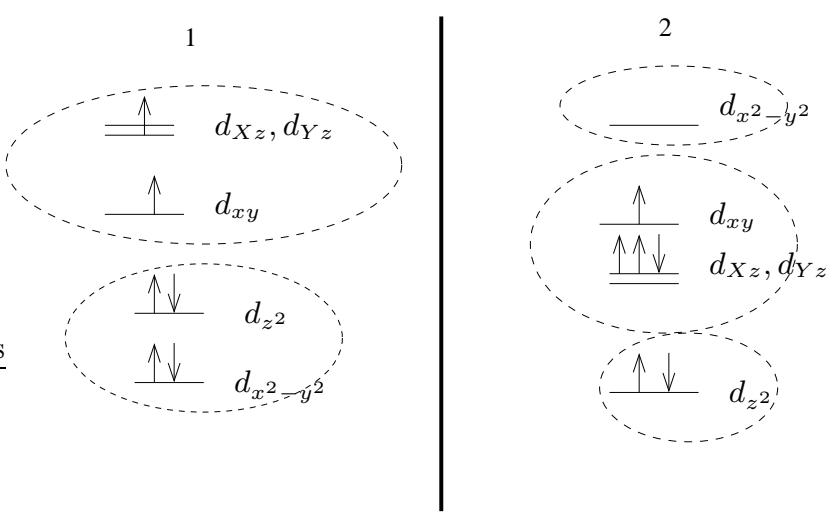

FIG. 2: Two scenarios for the level spacing of the iron atoms' d-orbitals which lead to orbital degeneracy and $S=1$. Each set of orbitals grouped together is assumed to fill up according to Hund's rule before any electrons are added to the next group of orbitals. This follows from assuming that the crystal field splitting between orbitals in different groups is greater than the Hund's coupling. Scenario 1 results in a single electron having to decide between two degenerate orbitals while Scenario 2 results in a single hole which is orbitally degenerate. We mainly discuss Scenario 1.

in this tetragonal structure are the $d_{x z}$ and $d_{y z}$ orbitals. Hence we will require an odd filling of this orbital pair. Two possible microscopic scenarios for the $d^{6}$ configuration of the $\mathrm{Fe}^{+2}$ ion are sketched in Figure 2, In the first, there is one electron available to occupy the two degenerate orbitals, while the orbital $d_{x y}$ is singly occupied. Interestingly, this is the orbital configuration suggested by the crystal field splitting of the Fe sites. For a perfect tetrahedral arrangement of Te ions, the $d_{x^{2}-y^{2}}$ and $d_{z^{2}}$ orbitals lie below the triplet of $d_{x y}, d_{x z}, d_{y z}$. The distortion of the tetrahedron in this material brings the $d_{x y}$ below the degenerate $d_{x z}, d_{y z}$ - leading to the orbital structure in Figure 2, Note, the sense of the distortion in FeTe is opposite to that in FeAs, where a similar exercise leads to a different orbital ordering ${ }^{3}$. Since such a local picture of electronic orbitals may not capture the physics of FeTe we also point out a different scenario (Figure 2 $\mathrm{b}$ ), where the orbital ordering is closer to what is predicted by the band structure calculations in $\mathrm{FeAs}_{\mathrm{s}} \frac{12}{2}$. If we order the dispersing bands by their center of mass, we end up with the ordering shown. Here too, the electron assignment can lead to a $S=1$ orbitally degenerate configuration, but now the degenerate pair of orbitals contains three electrons. We note that in both scenarios, the active orbitals are $d_{x y}, d_{x z}, d_{y z}$, which are also the ones expected to be present at the Fermi energy from weakly correlated band structure calculations of these materials 12,13 . The two scenarios are particle hole conjugates of one another, if one focuses on the active triplet of orbitals. Henceforth we will assume Scenario 1 for concreteness, as it corresponds better with experimental facts. The results there can be easily transcribed to Scenario 2, by a particle hole transformation.
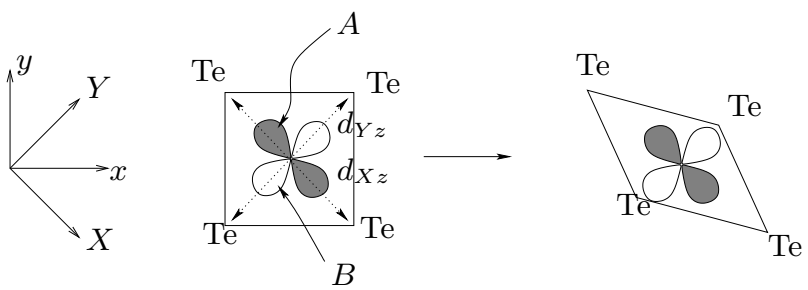

FIG. 3: Degeneracy lifting by Jahn-Teller distortion. The tellurium atoms projected into the $x y$-plane form a square in the undistorted compound, which distorts into a rhombus, so that $A>B$, lowering the energy of the shaded orbital.

\section{B. Orbital and Magnetic Order}

Let us discuss first the the pair of degenerate orbitals in Scenario 1, filled by a single electron. Later we will include the third orbital. It is convenient to rotate orbitals by $45^{\circ}$, and define another basis that point along the diagonals $d_{X z / Y z}=\frac{1}{\sqrt{2}}\left(d_{x z} \pm d_{y z}\right)$. Let $d_{1}^{\dagger}, d_{2}^{\dagger}$ be the creation operators for electrons in these diagonal orbitals. The Hamiltonian for this system is:

$$
H=H_{K E}+H_{U}+H_{J T}
$$

where the first term is the hopping hamiltonian, and the second and third terms refer to interactions and coupling to lattice phonons that lead to the Jahn-Teller effect.

$$
H_{U}=\sum_{r} \frac{1}{2} U\left(n_{r}-1\right) n_{r}-J_{H} \vec{S}_{1 r} \cdot \vec{S}_{2 r}
$$

where $n_{r}$ is the electron density at site $r$ and $\vec{S}_{a} r=$ $\frac{1}{2} d_{a r}^{\dagger} \vec{\sigma} d_{a r}$ is the spin on site $r$ in orbital $a$. In the limit of strong repulsion $U$ and a single electron per site, $\langle n\rangle=1$ we obtain an insulating state with orbital degeneracy. This degeneracy is typically resolved by the Jahn-Teller effect. A lattice distortion which breaks symmetry and splits the degeneracy occurs. The precise distortion that is realized is hard to predict, so we will assume that it is indeed of the type required to obtain the structural transition seen in this material. This involves a uniform orthorhombic distortion that changes the relative lengths of the two diagonal bonds. If we denote by $A$ and $B$, the classical bond lengths for the two diagonals (so $A=B$ in the tetragonal state), then we will assume that the lattice coupling is given by:

$$
H_{J T}=-\alpha(A-B) \sum_{r}\left(d_{1 r}^{\dagger} d_{1 r}-d_{2 r}^{\dagger} d_{2 r}\right)+\frac{\beta}{2}(A-B)^{2} .
$$

(See Fig. 3.) The orbital ordering implies that, in scenario 1 , the single electron on each site always occupies the same orbital, i.e., $n_{1 r}=1$ and $n_{2 r}=0$, or vice versa. For concreteness let us suppose that the $X$ diagonal expands. Then the electrons occupy orbital $1\left(d_{X z}\right)$ while orbital $2\left(d_{Y z}\right)$ is empty, because it is higher in energy. 
Since each site has an unpaired electron, we can now derive the Hamiltonian governing their magnetic moments.

In the insulating limit, the magnetic interaction is generated by virtual hopping of electrons. To proceed, we need to specify $H_{K E}$. Clearly, given the geometry of the $X z, /, Y z$ orbitals, hopping along the diagonals will be very anisotropic. We denote the $X(Y)$ diagonal hopping of the $d_{X z}\left(d_{Y z}\right)$ orbital by $t_{2}$, and let $t_{2}^{\prime}$ be the hopping of each in the orthogonal direction (see Fig. 4). The figure suggests that $t_{2} \gg t_{2}^{\prime}$ (see $\left.{ }^{14}\right)$, and for FeAs the nearest neighbor hoppings are comparatively small as well. Hence, we will simply work with the exchange interaction induced by $t_{2}$. Note, the next neighbor hopping only operates within a single sublattice (labeled $A$ and $B$ in the figure), so here we consider just the $A$ sublattice. The $t_{2}$ hopping will introduce antiferromagnetic exchange, but only along the $X$ diagonal $J_{2 X}=t_{2}^{2} / U$. This will lead to antiferromagnetic order along this diagonal direction. Note, assuming Scenario 1, this antiferromagnetic direction will be the expanded diagonal of the distorted compound, which is consistent with the observed wavenumbers of the magnetic and structural distortions ${ }^{3}$. Note, although the diagonal chains are ordered, there is negligible coupling between chains at this moment (because of the smallness of $t_{2}^{\prime}$ ). Below, we will see there is a more important mechanism that can lock the magnetic order in the chains together - the double exchange interaction.

\section{Double Exchange}

Hopping of electrons between the antiferromagnetic chains can readily occur if they occupy the $d_{Y z}$ orbital. However, in a orbitally ordered insulator, these are assumed to be empty. Given the empirical fact that FeTe is a metal, we assume a small occupation $\delta$ of electrons in this orbital. This can arise because of 'self-doping', i.e. if the orbital dispersion cannot be completely neglected, a fraction of carriers from one of the 'filled' bands (for example $d_{x y}$ ), could be transferred to an empty band, i.e. this orbital. This also leads to a conducting state at stoichiometry, as demanded by experimental observations. Furthermore, FeTe always occurs with a slight excess of Fe i.e. $\mathrm{Fe}_{1+y}$ Te. The electrons from the additional $\mathrm{Fe}^{+2}$ ions also contribute to $\delta$.

Such an excess carrier density will lead to ferromagnetic interactions between chains, via the double exchange mechanism. An electron hopping in this nearly empty orbital will be Hund's coupled to the $d_{X z}$ electron according to Equation 2. The Hund's coupling is typically large and will force both electrons to have the same spin. If the electron is to hop to the neighboring chain, the spins must be parallel. Then, it can enjoy a lowering of kinetic energy by $-2 t_{2}$. Thus, a ferromagnetic arrangement of spins will have a lower energy than an antiferromagnetic arrangement by an amount $2\left|t_{2}\right| \delta$, which can roughly be viewed as a ferromagnetic coupling along the $Y$ diagonal (strictly speaking this is a nonlocal interaction, and cannot be assigned solely to the diagonal bond). Thus $J_{2 Y}=-2\left|t_{2}\right| \delta$. With this combination of exchange constants, the magnetic ordering on a single sublattice is shown in Figure 4. Note that it has the wavevector $(\pi / 2, \pi / 2)$, as required.

\section{Coupling the Sublattices}

So far, the two sublattices $(A$ and $B)$ are independent. When the doping is small, the dominant spin interaction between nearest neighbor sites will arise from antiferromagnetic exchange from the electrons in the half filled $d_{x y}$ orbital. (The nearest neighbor hopping of electrons in the $d_{X z}$ orbitals would induce a smaller ferromagnetic interaction controlled by the Hund's coupling, since the dominant hopping is expected to switch the orbital index.) We will also invoke a coupling to the lattice to generate a biquadratic interaction term in order to lock in the commensurate wave number, leading to the net Hamiltonian describing the interaction of spin 1 atoms on a square lattice:

$$
H=\sum_{i j} J_{i j} \mathbf{S}_{i} \cdot \mathbf{S}_{j}-K \sum_{\langle i j\rangle}\left(\mathbf{S}_{i} \cdot \mathbf{S}_{j}\right)^{2}
$$

$J_{2 X}=t_{2}^{2} / U, J_{2 Y}=-2\left|t_{2}\right| \delta$ and $J_{1}>0$, and $J_{1}$ is the same for both nearest neighbor bonds. The phase diagram of this model as a function of increasing $K$ is included on the $y$-axis of Fig. 6. The $\mathbf{S}_{i} \cdot \mathbf{S}_{j}$ terms alone would lead to an incommensurate spiral state ${ }^{15}$, where the nearest neighbor spins in the spiral state are close to being orthogonal to one another (the "single spiral state" below). This state, even in the small incommensuration limit, is significantly different from the experimentally observed collinear state.

To stabilize the commensurate $\left(\frac{\pi}{2},-\frac{\pi}{2}\right)$ state, we take into account the biquadratic spin interaction $\left(-K\left(\mathbf{S}_{1}\right.\right.$. $\left.\mathbf{S}_{2}\right)^{2}$ ) which is a well-known consequence of spin-lattice coupling. This term prefers collinear magnetism. As we will see below, even modest values of the spin phonon coupling $K$ can induce locking of the commensurate, collinear phase observed in experiments. In particular, we show below that the critical coupling required to induce collinear order $K \gtrsim J_{1}^{2} / J_{2 X}$ can be parameterically smaller than $J_{1}$. Coupling of spin and lattice is presumably essential to getting a commensurate state at this wavevector.

Phase Diagram: The phase diagram can be obtained by taking the ansatz of a pair of coplanar spirals on the two sublattices with an arbitrary phase $\phi$ between them, and wavevector $k$ along diagonal $X$. This is probably sufficiently general to capture the ground states of Eqn. 4, because the ferromagnetic coupling along the $Y$-direction prevents the spin from varying in that direction. Let the azimuthal angle of spin $i$ be $\theta_{i}$. Then the ansatz reads:

$$
\theta_{i}=k\left(x_{i}-y_{i}\right)+(-1)^{x_{i}+y_{i}} \frac{\phi}{2} .
$$


a)

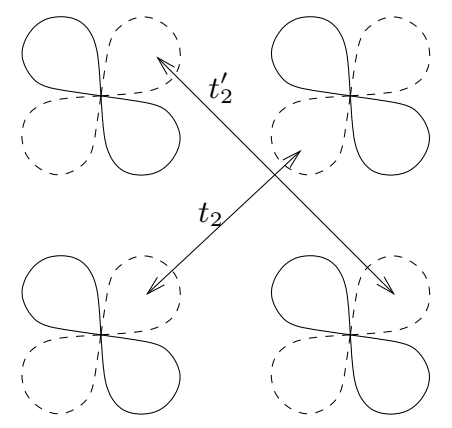

b)
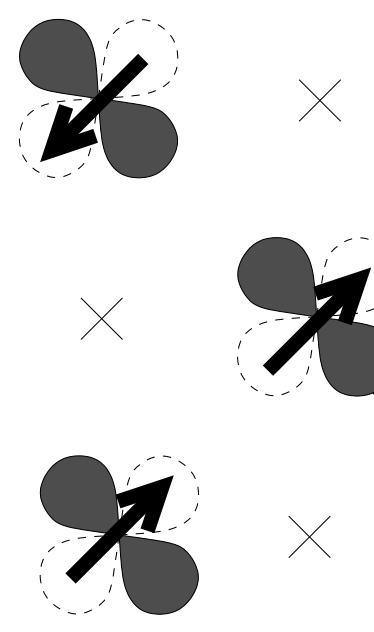
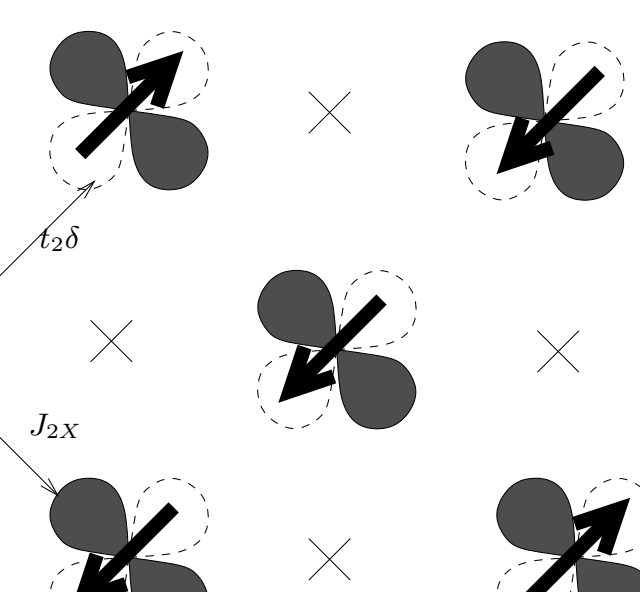

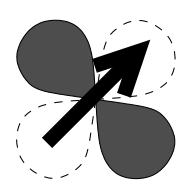

FIG. 4: How the spin-order on sublattices arises out of the orbital order. The solid and dotted lines show the projections of the $d_{X z}$ and $d_{Y z}$ orbitals. a) The second nearest neighbor hopping amplitudes, which determine the order in the sublattices. Electrons stay in the same orbital when they hop to the second nearest neighbor. The hopping for each orbital is anisotropic, with an anisotropy that depends on the orbital, so each orbital forms a set of one-dimensional chains. Electrons can hop between nearest neighbors as well (not shown). b) Generating the spin order in the A-sublattice. (The iron atoms in the B-sublattice are indicated by x's.) The $d_{X z}$ are shaded to indicate that they are filled and the $d_{Y z}$ orbitals are lightly doped. The $d_{X z}$ orbital form one-dimensional Mott insulators parallel to the $X$-axis with antiferromagnetic coupling $J_{2 X}$. The $d_{Y z}$ orbitals form one-dimensional metals parallel to the $Y$ direction. Ferromagnetic order along $Y$ lowers the kinetic energy of the metals by about $t_{2} \delta$.

Here, $(k,-k)$ is the wave number of the spin arrangement. (This state is ICA, from Ref. $\underline{6}$.) Note, the experimentally observed collinear state corresponds to $k=\pi / 2$ and $\phi=$ $\pi / 2$. Along any row, the angle between adjacent spins alternates between $k-\phi$ and $k+\phi$. For $k=\phi=\pi / 2$, the spins therefore alternate from parallel to antiparallel.

Minimizing the energy per site,

$$
\begin{aligned}
E[k, \phi]=J_{2 X} \cos 2 k+2 & J_{1} \cos k \cos \phi \\
& -K(1+\cos 2 k \cos 2 \phi)-2\left|t_{2}\right| \delta
\end{aligned}
$$

over $\phi$ and $k$ shows that there is a transition between a pure spiral with $\phi=0$ and incommensurate wavevector $k=\frac{\pi}{2}+O\left(\frac{J_{1}}{J_{2 X}}\right)$ and the collinear state $k=\pi / 2, \phi=\pi / 2$ when $4 K\left(J_{2 X}-K\right)=J_{1}^{2}$. For small $K \ll J_{2 X}$, the critical $K$ is $\approx J_{1}^{2} / 4 J_{2 X}$.

We can understand this result intuitively as follows. The spiral order occurs to lower the nearest neighbor energy. The coupling of a spin to its neighbors to the left and right cancels in the perfectly collinear state. To take advantage of $J_{1}$, the neighbors should therefore make an angle less than $180^{\circ}$, and the central spin should point opposite to the sum of the two neighboring spins. If $J_{1}$ is small compared to $J_{2 X}$, then the neighbors are nearly antiparallel, so $J_{1}$ has a very weak effect, explaining why the critical value for $K$ is not of order $J_{1}$ as one might have expected, but rather second order in $J_{1}$.

Spin Waves: To contrast this scenario with others that predict the same magnetic ordering pattern, we calcu- late the spin wave spectrum for the model 4 . We expect inelastic neutron scattering experiments in the future to be able to check this prediction. In particular, we contrast it with a recent theory $\underline{\underline{6} 16}$ in which there is a sufficiently large third neighbor exchange $J_{3}$ and the monoclinic distortion alters the first and second nearest neighbor interactions, leading to the observed magnetic order. In our model, the magnetic order is stabilized just by the anisotropy of $J_{2}$, with one ferromagnetic and one antiferromagnetic direction. (Ref ${ }^{6,16}$ also includes the anisotropic couplings but the third neighbor hopping makes it possible to stabilize the order without ferromagnetic directions.) Note, while doing the spin wave calculations, we expand about the equilibrium spin state, and hence the biquadratic interaction effectively leads to weak $(w)$ and strong $(s)$ nearest neighbor bonds, $J_{1 s} \neq J_{1 w}$. Thus, our spin wave dispersion is reproduced by a model involving only quadratic spin couplings, where $J_{1} \mathbf{S}_{1} \cdot \mathbf{S}_{2}-K_{1}\left(\mathbf{S}_{1} \cdot \mathbf{S}_{2}\right)^{2} \Rightarrow\left(J_{1}-2 K_{1}\left\langle\mathbf{S}_{1} \cdot \mathbf{S}_{2}\right\rangle\right) \mathbf{S}_{1} \cdot \mathbf{S}_{2}$ Therefore the bonds between parallel spins are effectively weaker than those between antiparallel spins, similar to Ref. $\underline{6.16}$. The spin wave spectrum is obtained using the Holstein-Primakoff expansion (see e.g.17). Figure 5 compares the dispersions one expects in the two models, the first with a strong $J_{3}$ and ours with a ferromagnetic diagonal coupling. Note, the upper band of the dispersions curves in the opposite direction along the $Y$-axis, because of the ferromagnetic coupling $J_{b}$. 

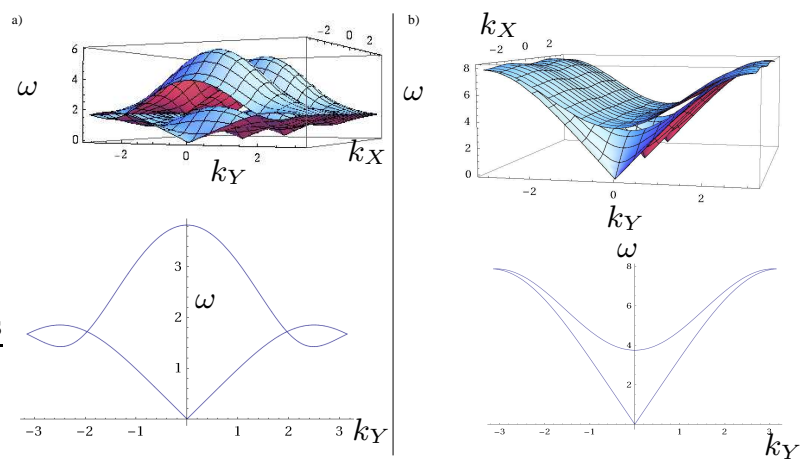

FIG. 5: Comparison of the spin wave dispersion for order stabilized by $J_{3}$ versus by a ferromagnetic diagonal interaction. The dispersions are plotted as a function of $\left(k_{X}, k_{Y}\right)=$ $\left(k_{x}-k_{y}, k_{x}+k_{y}\right)$ in the upper pictures. The other graphs show the dispersion in the $Y$ direction. a) The spin wave spectrum for $J_{2 X}=J_{2 Y}=1, J_{1}=1.47, K=0.265, J_{3}=0.4$, which have the same effective $J_{1 w, s}$ as in Ref.6. b) This shows the spectrum for $J_{2 X}=-J_{2 Y}=1, J_{1}=0.7, K=0.265, J_{3}=0$. The sign of $J_{2 Y}$ is flipped to describe a ferromagnetic coupling, while $J_{3}$ is set to zero. ( $J_{1}$ 's value is decreased relative to a) to stabilize the order.) Note that the upper band changes from a hill shape to a valley.

\section{E. Doping Induced Incommensuration}

The properties of $\mathrm{Fe}_{1+y} \mathrm{Te}$ have been experimentally investigated as $y$ is varied. Experimentally, $\mathrm{Fe}_{1+y} \mathrm{Te}$ is found to have incommensurate spin order when $y$ is large enough, with an incommensurate wavevector that deviates from $(\pi / 2, \pi / 2)$ linearly with doping ${ }^{3}$. One of the effects of the excess iron, which is believed to be in the $\mathrm{Fe}^{2+}$ state, is to electron dope the system by $2 y$ electrons.Here we consider how this may be explained as a result of the increasing electron density $\delta=2 y$ in the $Y z$ orbital, which makes nearest neighbor hopping more important. This will lead to incommensurate order that is related to the doping level.

To see this we will consider two dimensional motion of the doped electrons, which can occur only if hopping amplitudes other than $t_{2}$ are taken into account. These types of motion are limited by the wide stripes of the commensurate $\left(\frac{\pi}{2}, \frac{\pi}{2}\right)$ spin pattern, increasing the kinetic energy, according to the uncertainty principle. Such kinetic energy effect has a stronger dependence on the relative angle between a pair of spins when the angle is close to $90^{\circ}$ than the interactions considered above, so it is able to distort the commensurate collinear state with wave vector $\left(\frac{\pi}{2},-\frac{\pi}{2}\right)$ and $\phi=\pi / 2$, into a "double spiral", a slightly twisted version of the same state, of the form Eq. (5), and with $\phi \approx \frac{\pi}{2}$ and $k \approx \frac{\pi}{2}$, even when the doping is weak.

To understand the kinetic energy effect, we assume a uniformly varying classical spin configuration and minimize the energy of a single Hund's coupled electron, Eq. (2), hopping in this background. The resulting kinetic en- ergy $\left(K E_{\min }\right)$ times electron density, $K E_{\min } \delta$ is added to the magnetic energy (4), and this total energy is minimized to obtain the phase diagram in Fig. 6] A shortcoming of this phase diagram is that the spiral phases may not be stable against phase separation (see below).

We will now focus for simplicity on determining the kinetic energy due to hopping via $t_{2}^{\prime}$. We checked also that the nearest neighbor $t_{1}$ of Ref $\underline{\underline{14}}$ leads to a very similar phase diagram. The kinetic energy is computed from the hopping Hamiltonian $H_{t_{2}^{\prime}}=-t_{2}^{\prime} \sum_{i} d_{Y z}^{\dagger}\left(\mathbf{r}_{i}+\hat{\mathbf{x}}-\right.$ $\hat{\mathbf{y}}) d_{Y z}\left(\mathbf{r}_{i}\right)+$ h.c., which favors ferromagnetic alignment along the $X$-diagonal.Since the Hund's energy is larger than the $t$ 's, the natural basis for spin states on each site are the states quantized along the local spin orientation of the magnet $\left(\theta_{i}\right)$. If the Hund's energy is assumed to be infinite, we can assume that the electron state is always aligned with the local spin. Such a state, with momentum $\mathbf{p}$ is represented by the following electron wavefunction,

$$
\psi_{i}=\frac{1}{\sqrt{2}}\left(\begin{array}{c}
e^{-i \frac{\theta_{i}}{2}} \\
e^{i \frac{\theta_{i}}{2}}
\end{array}\right) e^{i \mathbf{p} \cdot \mathbf{r}_{i}} .
$$

We can now find the variational energy of this wave function, and minimize with respect to $p$.

The expectation value of the kinetic energy for a bond between a pair of sites is $2 t \Re\left(\psi_{i}^{\dagger} \psi_{j}\right)$. Aside from the phase factor from the momentum, this overlap is proportional to $\cos k$ for spins adjacent along the $X$ direction (the cosine of half the angle between the spins). In particular, because the spin is conserved during the hopping, the electron cannot hop at all onto a site with an antiparallel spin. The electron kinetic energy is $2 t_{2} \cos \left(p_{x}+p_{y}\right)+2 t_{2}^{\prime} \cos \left(\frac{p_{x}-p_{y}}{2}\right) \cos k$. Minimizing over $p$, we obtain $K E_{\text {min }}=-2\left|t_{2}\right|-2\left|t_{2}^{\prime} \cos (k)\right|$. Combining this with the previously obtained energy in the absence of $t_{2}^{\prime}$, Eqn. (6), and minimizing with respect to $\phi$ and $k$ we obtain the double spiral. This can be easily seen when the doping is very weak: focusing on the competing terms $J_{2 X}$ and $t_{2}^{\prime} \delta$, and assuming $k \approx \frac{\pi}{2}$ to find $E=J_{2 X} \cos (2 k)-2 t_{2}^{\prime}|\cos k| \approx-J_{2 X}+2 J_{2 X}(k-$ $\left.\frac{\pi}{2}\right)^{2}-2 t_{2}^{\prime} \delta\left|k-\frac{\pi}{2}\right|$, so $\left|k-\frac{\pi}{2}\right|=\frac{t_{2}^{\prime} \delta}{2 J_{2 X}}$ minimizes the energy. The incommensuration therefore is proportional to the doping strength, as observed experimentally.

If we allow the electrons to hop virtually into states that violate Hund's rule, the commensurate state survives over a finite range of dopings. When the twisting is very small, it becomes easier for an electron that is trying to hop along the antiferromagnetic diagonal to hop through the Hund's violating states than to stay in states that are parallel to the local spin but which have very small overlaps. In this case, there is no reason for the spin order to distort at all. The virtual hopping has the greater efficiency when $\left|t_{2}^{\prime} \cos k\right|<\left|\frac{t_{2}^{\prime 2}}{J_{H}}\right|$, or (using the result for $k$ at small $\delta$ ), $\delta \lesssim \frac{J_{2 X}}{J_{H}}$. (A more detailed calculation gives the same result.) Quantum fluctuations also allow the electrons to hop more easily in this direction, by temporarily making adjacent atoms become oriented parallel to one another. 

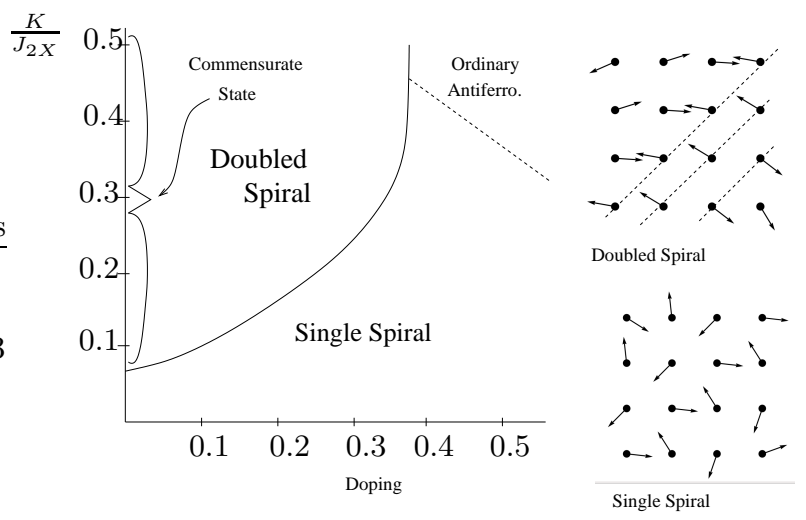

FIG. 6: The phase diagram as a function of $K$ and doping for $J_{1}=\frac{1}{2} J_{2 X}, t_{2}^{\prime}=1.5 J_{2 X}$, using the infinite Hund's coupling approximation. The three phases are the doubled spiral, the single spiral, and ordinary $(\pi, \pi)$ antiferromagnetism. Note that both the single and doubled spirals are constant along wavefronts parallel to $Y$. In the perpendicular direction, the double spiral alternates between rotating through small angle and angles close to $180^{\circ}$, while the single spiral rotates through around $90^{\circ}$ each time. A calculation which takes fluctuations or the finiteness of the Hund's constant into account would find a finite range of dopings where the commensurate phase is stable. The solid/dashed line represents a discontinuous/continuous transition. The discontinuous transition boundary should actually be replaced by a wide swath of phase separation.

A very similar phase diagram appears for $t_{1}$ hopping. This hopping probably has weaker effects, however, since the orbital switches with each hop. This implies that an electron alternates between Hund's rule violating and satisfying states, so that the amplitude for a pair of these steps is of order $\frac{t_{1}^{2}}{J_{H}}$. This process leads to an antiferromagnetic interaction along rows and columns, but this causes incommensuration just as the ferromagnetism induced by motion along the diagonals did.

Although these calculations explain how incommensurate order can occur, the order differs from the experimentally proposed pattern in $\underline{3}$. The proposed order has the spin dominantly along the $\hat{Y}$ direction whose magnitude is modulated with an incommensurate wavevector, and also a spin spiral composed of the orthogonal spin directions, at the same wavevector. While further experimental work is required to confirm the true nature of the complex incommensurate order, we note one interesting measurement, that orthorhombic symmetry is recovered at higher doping, (eg. at $x=0.141^{3}$ ) where the incommensurate state is stabilized. This is consistent with our assumption that the monoclinic part of the distortion is strongly coupled to spin order. Fig. 1 shows that the sense of the monoclinic distortion is correlated with the bond energies $\vec{S}_{i} \cdot \vec{S}_{j}$. Shifting the spin ordering and thus the pattern of bond energies over one site would cause the lattice to tilt in the opposite direction along the $Y$ axis. For an incommensurate or-

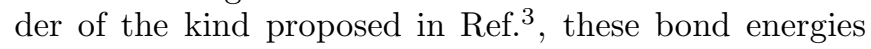

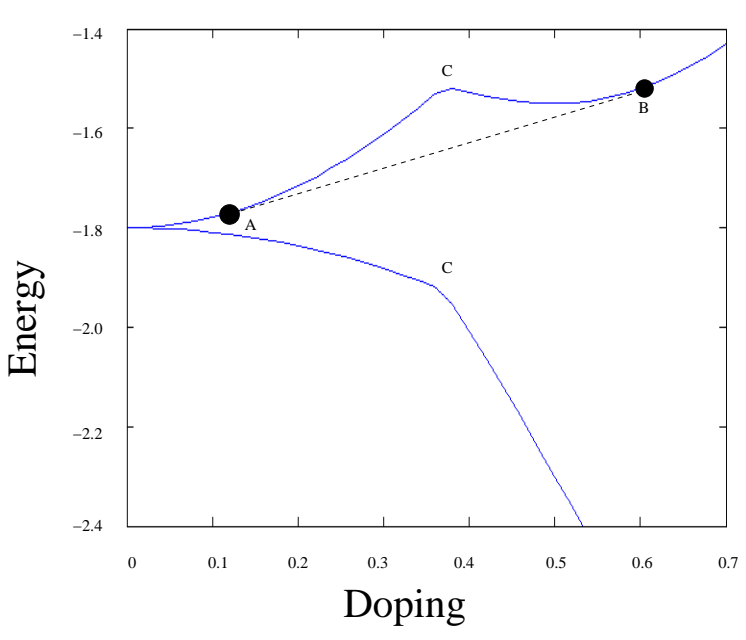

FIG. 7: Energetics of phase separation. The solid curve shows the energy of states with uniform doping, with repulsion $V=$ $6 J_{2 X}$ (upper curve) and without. The parameters are the same as in Fig. 6. with $K=0.4$. The concave down portion of each energy curve is unstable to phase separation. The points labelled $C$ show the unphysical transition between the doubled- and single-spiral states. The dashed line corrects the upper energy-curve, by showing the energy of the state where the $A$ and $B$ phases coexist.

are also modulated with an incommensurate wavevector which removes the monoclinic distortion. In contrast, for the orders described by Eqn 5 including both the double and single spirals we have considered, the bond energies are independent of incommensuration, hence a monoclinic distortion is expected throughout. An example of a spiral order that would not induce a monoclinic distortion, close to the commensurate state of interest is: $\theta_{i}=(-1)^{x_{i}+y_{i}} k\left(x_{i}-y_{i}\right)$, with $k$ close to $\frac{\pi}{2}$. Effects that we have neglected, including spin anisotropy and the effect of the excess iron moments, can modify the precise form of the incommensurate state.

Phase Separation An additional shortcoming of this explanation of the incommensurate order is that the spiral phases can be unstable to phase separation $\frac{18}{}$. The doped electrons prefer to be segregated into high and low density regions, with different spin orders. The predictions above can still be relevant, because repulsive forces between electrons help to limit the phase separation.

Fig. 7 shows the energy as a function of doping both without and with a strong short-range repulsion, described by adding a term $\frac{1}{2} V \delta^{2}$ to the energy functional. In the figure, $K=0.4$ and the other parameter values taken from Fig. 6, A concave down portion is unstable to phase separation. For a doping in between $A$ and $B$, i.e., $0.1 \leq \delta \leq 0.6$, dividing the compound up into two regions with the dopings 0.1 and 0.6 gives a state with a lower energy, whose energy is represented by the tangent. In this case the phase mixture is between a doubled spiral with a low electron density and an antiferromagnetically ordered high density portion. 
The Coulomb interactions can have one of two consequences; they either stabilize a uniform state as just described (if the force is strong enough at short distances), or else they force the two coexisting phases to fill small alternating portions of the $\mathrm{FeTe}$, rather than becoming completely segregated. The ordering with alternating regions of the $\left(\frac{\pi}{2}, \frac{\pi}{2}\right)$ and $(\pi, \pi)$ phases could also be incommensurate.

Additional Doping A surprising order may arise if the compound is sufficiently doped, which directly connects to our central assumption that the orthorhombic part of the structural distortion arises from orbital, rather than spin ordering. In our model, large electron doping would drive ferromagnetic order on each of the A and B sublattices. This optimizes the electron kinetic energy. The sublattices continue to couple together antiferromagnetically, leading to $(\pi, \pi)$ magnetic order. In Fig. 6] the order varies from commensurate, to the rhythmic spiral phase to the $(\pi, \pi)$ antiferromagnetic phase as the doping increases along a line at $K=0.5 J_{2 X}$. For a smaller value of $K$, the pure spiral phase occurs before $(\pi, \pi)$ antiferromagnetism sets in. Note however, that while $(\pi, \pi)$ antiferromagnetic order is compatible with tetragonal symmetry, here we expect orbital order to persist and result in an orthorhombic distortion, clearly signaling the independence of structural and magnetic order.

On the other hand, hole doping weakens the kinetic energy effect to the point where each sublattice has ordinary antiferromagnetic order, and the biquadratic interaction orients the two sublattice-spins parallel to each other, leading to $(\pi, 0)$ magnetic order of the type seen in the FeAs materials. Again, while the $(\pi, 0)$ ordering would be accompanied by compression along the $x$ or $y$ axis, if this were magnetically driven, here we expect the orbital ordering to remain the same, so the diagonal distortion would not change. A more direct test of whether the distortion or the magnetic ordering is the primary phenomenon is to apply a strong magnetic field to eliminate the magnetic ordering and see whether the distortion remains. This would be possible if the exchange interactions are weak, or can be weakened by modifying the compound somehow, e.g., by applying pressure.

\section{TRANSPORT AND SINGLE ELECTRON PROPERTIES}

The main consequence for transport of the orbital ordering induced quasi one dimensionality is that the conductivity should be strongly anisotropic. Below the orbital and magnetic ordering temperature, the excess electrons in the $Y z$ band move much more readily in the $Y$ direction, because $t_{2}$ is greater than $t_{1}, t_{2}^{\prime}$. Furthermore, the spins are ferromagnetically aligned along this direction, and can hence propagate easily. To travel in the orthogonal direction, they must cross through diagonals where the spins are oppositely aligned. While a difference in electrical conductivity along the two diag- onal directions is hardly surprising given the symmetry of the low temperature state, the specific prediction here is that this will be a significant effect, and the nature of the anisotropy is that the low conductance is to be found along the antiferromagnetically ordered diagonals.

Experimentally, a Drude peak has been observed to develop below the ordering transition ${ }^{5}$. Since a test of anisotropy demands a single domain, optical conductivity on a sample where the domain size is larger than the spot size is required, and should be feasible. There, depending on the direction of the polarization, a different conductivity should result. Note, writing $\sigma(\omega)=\frac{n e^{2} \tau}{m^{*}} \frac{1}{1+i \omega \tau}$, both the effective mass $m^{*}$ and the scattering rate $\tau$ are expected to be anisotropic, which can be separately inferred from a knowledge of $\sigma(\omega)$.The anisotropy in this scenario is expected to be particularly prominent in the low frequency limit, since scattering by spins will limit the scattering time along the antiferromagnetic diagonal. At higher frequencies, only the anisotropy of $m^{*}$ will have an effect. However, if the scattering rate for the $X$ direction is extremely large, $\sigma(\omega)$ will be flat in that direction, while conductivity along $Y$ will have a Drude peak.

$A R P E S$ Given the quasi one-dimensional dispersion along the diagonals, a narrow elliptical Fermi surface tilted at $45^{\circ}$ to the Fe-Fe bonds, is expected to appear below the ordering temperature. Moreover, these would be orbitally polarized, which can be experimentally tested using polarized light to determine orbital content along high symmetry directions. When the scattering plane containing the photon and the ejected electron is perpendicular to the surface, and along, say, the $X z$ plane, selection rules imply that $S(P)$ polarized light with polarization perpendicular to (parallel to) the scattering plane, ejects only electrons in the $Y z(X z)$ orbital. The strongly dispersing direction should therefore appear for $S$ polarized light, and will have the form shown in Figure 8. if a single domain is imaged. The polarization dependence provides an experimental signature even from multi-domain data. Current ARPES data on $\mathrm{FeTe}^{4}$ has not reported such a signature, however, the intensity associated with this Fermi surface segment is hard to estimate reliably. Direct experimental probes of orbital ordering should also be able to test this scenario. We hope this prediction will stimulate further experiments.

\section{CONCLUSIONS}

We have argued that FeTe, a material closely related to the recently discovered Fe based superconductors, is likely to be a fairly strongly correlated material. This motivates us to use a local picture of the electronic structure, which in turn can explain, quite naturally, the unusual magnetism observed in this material. The key ingredient of this scenario is an emergent quasi one dimensionality arising from orbital ordering along the diagonals. While this theoretical scenario is a simplified caricature of the real system, it does make several qualitative predictions 


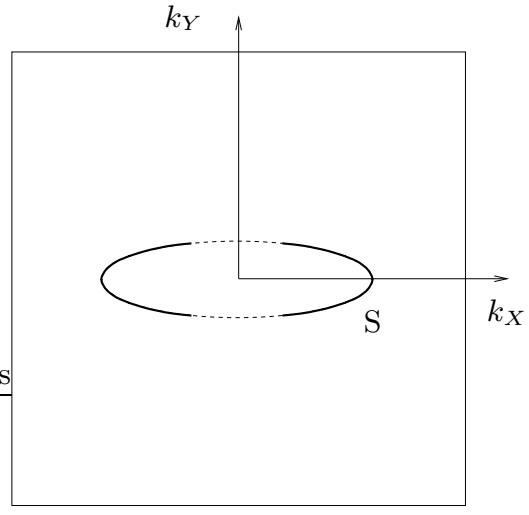

FIG. 8: The shape of the Fermi surface of the $Y z$ electrons in the actual Brillouin zone. We predict that the surface is narrow in the ferromagnetic direction. Furthermore, certain parts of the ellipse should disappear in ARPES with polarized light. The dashed portions of the Fermi surface fade out for $S$ polarization. This tests the assumption that the anisotropy results not from the lattice distortion but because the dispersing electrons are in a single orbital. for experiments which should be readily testable. The conductivity should be anisotropic, with higher conductivity along the ferromagnetic direction, if the unusual magnetic order is caused by the kinetic energy of conduction electrons. Certain parts of the electron Fermi surface should disappear in polarized ARPES, indicating the orbital ordering. Lastly, if the Jahn-Teller effect causes the orthorhombic distortion, then the distortion should persist even when the magnetic order is removed or changed to one which would not be expected to favor lattice distortion on the basis of symmetry.

We thank Eugene Demler for thoughtful conversations, and acknowledge support from LBNL DOE-504108.
1 Y. Kamihara, T. Watanabe, M. Hirano, and H. Hosono, J. Am. Chem. Soc. 130, 3296 (2008).

2 W. Z. Hu, J. Dong, G. Li, Z. Li, P. Zheng, G. F. Chen, and J. L. Luo, Phys. Rev. Lett. 101, 257005 (2008).

3 W. Bao, Y. Qiu, Q. Huang, M. A. Green, P. Zajdel, M. R. Fitzsimmons, M. Zhernenkov, M. Fang, B. Qian, E. K. Vehstedt, et al. (2008), arxiv:0809.2058.

${ }^{4}$ Y. Xia, D. Qian, L. Wray, D. Hsieh, G. F. Chen, J. L. Luo, N. L. Wang, and M. Z. Hasan (2009), arXiv:0901.1299.

${ }^{5}$ G. F. Chen, Z. G. Chen, J. Dong, W. Z. Hu, G. Li, X. D. Zhang, P. Zheng, J. L. Luo, and N. L. Wang (2008), arXiv:0811.1489.

${ }^{6}$ C. Fang, B. A. Bernevig, and J. Hu (2008), arxiv:0811.1294.

7 M. D. Johannes and I. Mazin, Phys. Rev. B (2009), arXiv:0904.3857.

8 M. J. Han and S. Y. Savrasov (2009), arxiv:0903.2896.

9 F. Krüger, S. Kumar, J. Zaanen, and J. van den Brink,
Phys. Rev. B 79, 054504 (2009).

10 R. R. P. Singh (2009), arXiv:0903.4408.

11 W. Lv, J. Wu, and P. Phillips (2009), arXiv:0905.1704.

12 W. Malaeb, T. Yoshida, T. Kataoka, A. Fujimori, M. Kubota, K. Ono, H. Usui, K. Kuroki, R. Arita, H. Aoki, et al., J. Phys. Soc. Jpn. 77, 093714 (2008).

13 P. A. Lee and X. G. Wen, Phys. Rev. B 78, 144517 (2008).

14 Y. Ran, F. Wang, H. Zhai, A. Vishwanath, and D.-H. Lee, Phys. Rev. B 79, 014505 (2009).

15 C. Xu and J. Hu (2009), arXiv:0903.4477.

16 F. Ma, W. Ji, J. Hu, Z.-Y. Lu, and T. Xiang, Phys. Rev. Lett. 102, 177003 (2009).

17 O. Madelung, Introduction to Solid-State Theory (Springer-Verlag, Berlin, 1996).

18 E. Dagotto, T. Hotta, and A. Moreo, Physics Reports 344, 1 (2001). 